\title{
Low Temperature Electron Microscopy of "Charge-Ordered" Phases
}

Ismail El Baggari ${ }^{1 *}$, David J. Baek ${ }^{2}$, Benjamin H. Savitzky ${ }^{1}$, Michael J. Zachman ${ }^{3}$, Robert Hovden ${ }^{4}$ and Lena F. Kourkoutis ${ }^{3,5^{*}}$

1. Department of Physics, Cornell University, Ithaca, NY, USA.

2. School of Electrical and Computer Engineering, Cornell University, Ithaca, NY, USA.

3. School of Applied and Engineering Physics, Cornell University, Ithaca, NY, USA.

4. Department of Materials Science \& Engineering, University of Michigan, Ann Arbor, MI, USA.

5. Kavli Institute at Cornell for Nanoscale Science, Cornell University, Ithaca, NY, USA.

* Corresponding authors: ie52@cornell.edu \& lena.f.kourkoutis@cornell.edu

Scanning transmission electron microscopy (STEM) is highly sensitive to lattice degrees of freedom, enabling tracking of complex lattice displacements with sub-Angstrom resolution and picometer precision [1]. Due to tight stability tolerances, most STEM measurements are done at room temperature but as shown recently, cryogenic STEM near liquid nitrogen temperature is possible using available instrumentation and image stack alignment $[2,3]$. This paves the way to mapping subtle lattice and electronic orders which emerge at low temperature.

Charge-ordered phases are noteworthy for their ubiquity across material systems and their diverse electronic effects which include competition with superconductivity, metal-insulator transitions, or spin and orbital ordering. Visualizing the electronic component of charge-ordered states at the atomic scale is a demonstrably powerful approach to understand their origin and behavior [4]. The role of the lattice, however, is comparatively less explored, despite being tied to complex spin/orbital textures. Here, we use cryogenic STEM to visualize the nature of charge-ordered phases at low temperature.

Figures 1(a) and (b) show STEM images of the layered 1T'- $\mathrm{TaTe}_{2}$ at $293 \mathrm{~K}$ and $93 \mathrm{~K}$, respectively. In addition to the presence of a lattice distortion at room temperature, this material undergoes a structural transition at $\sim 170 \mathrm{~K}$ due to polymerization of Ta sites, with conflicting reports of both dimerization and trimerization [5]. The transformation is accompanied by an unusual transition to a more metallic state. The amplitude of the Fourier transform at 93 K (Fig. 1(d)) reveals superlattice peaks consistent with a threefold, hexagonal superstructure. By analyzing subtle intensity modulations in STEM data (Fig. 1(c)), we find that the low temperature phase exhibits longitudinal Ta displacements along the b-axis which are consistent with trimer states.

In manganites, the structure of charge order has been debated extensively since bulk measurements are consistent with multiple metastable structures. Figure 2(a) shows the periodic lattice displacement (PLD) pattern at $93 \mathrm{~K}$ in $\mathrm{Bi}_{0.35} \mathrm{Sr}_{0.2} \mathrm{Ca}_{0.45} \mathrm{MnO}_{3}$, furnishing real space evidence for the so-called Wigner-crystal model [6]. In half-doped compounds, the charge order structure is also actively contested, with theoretical and experimental evidence for both a stripe structure and a bi-stripe structure [7]. Figure 2(b) shows the PLD pattern in a $\mathrm{Nd}_{1 / 2} \mathrm{Sr}_{1 / 2} \mathrm{MnO}_{3}$ thin film which is consistent with the stripe phase. Remarkably, this state is inhomogeneous over tens of nanometers and is interspersed by regions of bi-stripe order (Fig. 2(c)). As both these phases have the same periodicity and are disrupted by nanoscale disorder, real space visualization is necessary to map unconventional lattice behavior and discriminate between microscopic models of charge and orbital order [8].

References:

[1] A. B. Yankovich et al., Nat. Commun. 5 (2014), p. 4155.

[2] I. El Baggari et al., Proc. Natl. Acad. Sci. 115, 1445 (2018), p. 1445-1450.

[3] B. H. Savitzky et al., Ultramicroscopy 191 (2018), p. 56-65. 
[4] A. Soumyanarayanan et al., Proc. Natl. Acad. Sci. 110 (2013), p. 1623-1627.

[5] J. Feng et al., Appl. Phys. Lett. 109 (2016), 021901.

[6] P. G. Radaelli, et al., Phys. Rev. B 59 (1999), p. 14440.

[7] R. J. Goff, J. P. Attfield, Phys. Rev. B 70 (2004), p. 140404.

[8] This work was supported by AFOSR (FA 9550-16-1-0305), NSF (DMR-1539918, DMR-1429155, DMR-1719875), and the Packard Foundation.
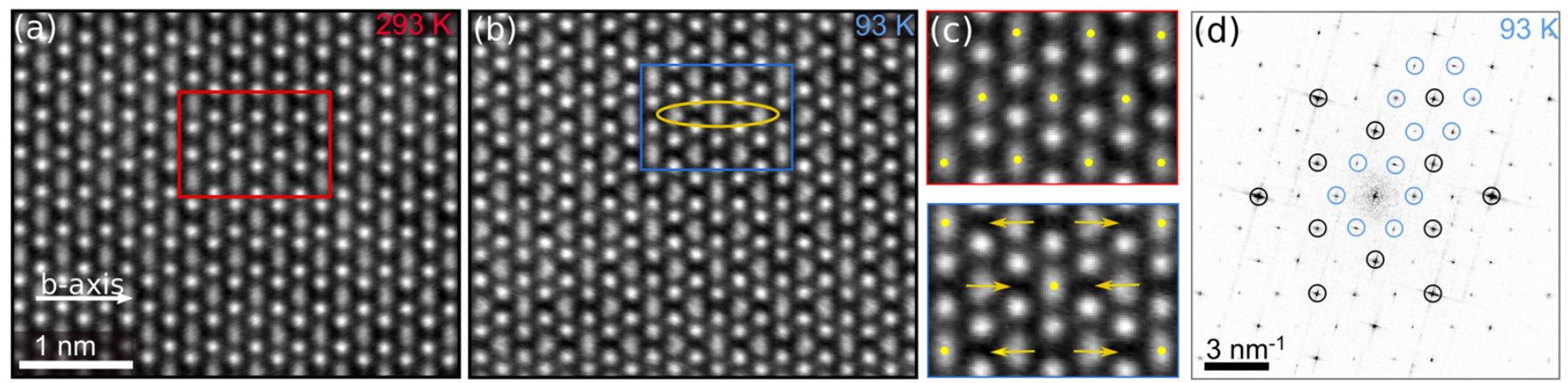

Figure 1. Low temperature Ta trimers in 1T'-TaTe2. (a), (b) STEM images of $1 \mathrm{~T}^{\prime}-\mathrm{TaTe}_{2}$ at $293 \mathrm{~K}$ and $93 \mathrm{~K}$, respectively. The low temperature phase exhibits Ta displacements forming trimer states (ellipse). (c) Zoom-in of red box (top) showing elongated Ta columns (yellow dots) due to the room temperature distortion. Tellurium columns appear round. At low temperature (bottom), further distortions of Ta column shapes indicate longitudinal displacements (arrows) of Ta atoms. (c) Fourier transform amplitude of a $15 \mathrm{~nm}$ field-of-view STEM image of $1 \mathrm{~T}^{\prime}-\mathrm{TaTe}_{2}$ at $93 \mathrm{~K}$. Superlattice peaks (blue circles) emerge in the low temperature phase.
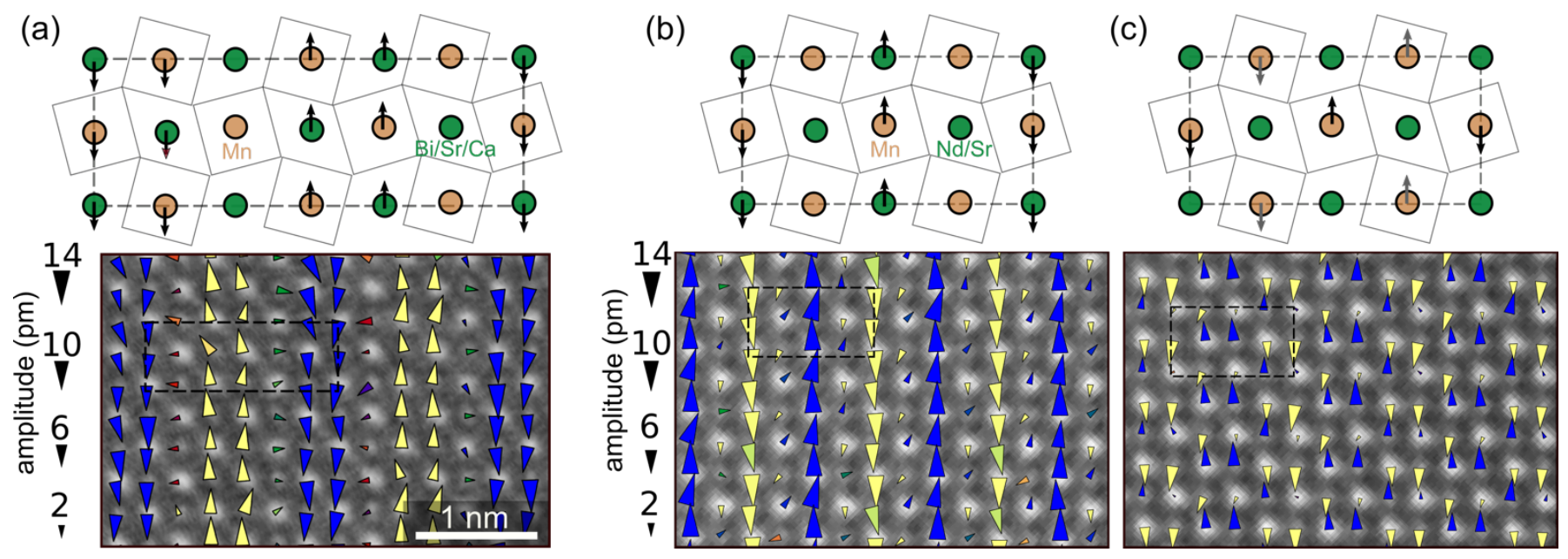

Figure 2. Visualizing the microscopic structure of charge-ordered manganites. (a) Cryogenic STEM imaging of $\mathrm{Bi}_{0.35} \mathrm{Sr}_{0.2} \mathrm{Ca}_{0.45} \mathrm{MnO}_{3}$ and periodic lattice displacements (PLD) of cations in the chargeordered phase. Arrows indicate the direction of the displacements, with the area scaling with the displacement amplitude. The pattern in consistent with the Wigner-crystal model. (b) PLD map in the half-doped $\mathrm{Nd}_{1 / 2} \mathrm{Sr}_{1 / 2} \mathrm{MnO}_{3}$ thin film. The structure is consistent with the so-called stripe phase. (c) PLD map in a different region showing a more complicated structure consistent with the bi-stripe phase. 